\title{
Compressible Kelvin-Helmholtz Instability at the Terrestrial Magnetopause
}

\author{
Alejandro G. González* \\ Instituto de Física Arroyo Seco, Universidad Nacional del Centro de la \\ Provincia de Buenos Aires, Pinto 399, 7000 Tandil, Buenos Aires, Argentina \\ Julio Gratton, Fausto T. Gratton*, \\ INFIP Instituto de Física del Plasma, Facultad de Ciencias Exactas y Naturales, \\ Universidad de Buenos Aires, Pabellón I, Ciudad Universitaria, 1428, Buenos Aires, Argentina \\ and Charles J. Farrugia \\ Space Science Center, University of New Hampshire at Durham, NH, USA.
}

Received on 30 June, 2002

\begin{abstract}
The compressible magnetohydrodynamic Kelvin-Helmholtz instability occurs in two varieties, one that can be called incompressible as it exists in the limit of vanishing compressibility (primary instability), while the other exists only when compressibility is included in the model (secondary instability). In previous work we developed techniques to investigate the stability of a surface of discontinuity between two different uniform flows. Our treatment includes arbitrary jumps of the velocity and magnetic fields as well as of density and temperature, with no restriction on the wave vector of the modes. Then it allows stability analyses of complex configurations not previously studied in detail. Here we apply our methods to investigate the stability of various typical situations occurring at different regions of the front side, and the near flanks of the magnetopause. The physical conditions of the vector and scalar fields that characterize the equilibrium interface at the positions considered are obtained both from experimental data and from results of simulation codes of the magnetosheath available in the literature. We give particular attention to the compressible modes in configurations in which the incompressible modes are stabilized by the magnetic shear. For configurations of the front of the magnetopause, which have small relative velocities, we find that the incompressible MHD model gives reliable estimates of their stability, and compressibility effects do not introduce significant changes. However, at the flanks of the magnetopause the occurrence of the secondary instability and the shift of the boundary of the primary instability play an important role. Consequently, configurations that are stable if compressibility is neglected turn out to be unstable when it is considered and the stability properties are quite sensitive on the values of the parameters. Then compressibility should be taken into account when assessing the stability properties of these configurations, since the estimates based on incompressible MHD may be misleading. A careful analysis is required in each case, since no simple rule of thumb can be given.
\end{abstract}

\section{Introduction}

The Kelvin-Helmholtz Instability (KHI) can occur in laboratory and astrophysical plasmas when two (or more) regions are in relative motion. Other properties of the plasma (like magnetic field, density and temperature) may also change across the transition layer. These changes modify the stability and other properties of the modes, thus complicating analysis. The KHI may occur at the terrestrial magnetopause, the boundary that separates the interplanetary magnetic field (IMF) present in the magnetosheath, and the geomagnetic field inside the magnetosphere. The relative motion is generated by the solar wind flowing through the magnetosheath. The flow velocity and the magnetic field are assumed parallel to the transition layer, and the magnetic field may change its direction (magnetic shear) and its magnitude across the transition. The low frequency magnetohydrodynamic (MHD) modes shall be our main concern here since their instability may produce large-scale turbulence. The KHI is considered a major source of

\footnotetext{
${ }^{*}$ Researcher of the Consejo Nacional de Investigaciones Científicas y Tecnológicas
} 
anomalous transport of momentum from the solar wind into the magnetosphere, and a cause of viscous drag at the magnetopause. This is so particularly during periods of northward IMF, when the reconnection of magnetic lines is less likely to occur. We shall not consider other unstable modes that can also arise close to, and inside the magnetopause, in frequency ranges higher than those treated by MHD. These modes lead to microscopic turbulence that affects the transport properties of the plasma at smaller wavelengths. We shall also neglect dissipative effects so that our treatment shall be based on ideal MHD. The KHI at the magnetopause has been examined since the 1950's, and a large literature has grown on this subject. We quote here some recent reviews [?], [?], [?] among several others.

The theory of the KHI for arbitrary geometry is exceedingly complicated, but fortunately in many circumstances, the wavelength of the perturbation is very small as compared to the curvature radii of the transition layer. Then the problem can be treated in a plane slab geometry, in which the unperturbed quantities (density $\rho$, pressure $p$, magnetic field $\mathbf{B}$ and mass flow velocity $\mathbf{u}$ ) depend only on the $y$ coordinate (perpendicular to the transition layer). A differential equation for the linear MHD perturbations of stratified plasmas [?], and its generalization to include the effect of gravity [?], can be used as a starting point to investigate these problems.

If the wavelength of the perturbation is large as compared to the thickness of the transition layer, we can ignore the structure of the transition and assume it is a mathematical surface (at $y=0$ ) separating two infinite, uniform plasma regions. The problem can then be treated by means of standard normal mode analysis. The equations of ideal MHD and the parameters of the configuration do not involve either a frequency or a length scale. Therefore the theory only predicts the (complex) phase velocity $v$ of the perturbation $\left(v=\omega / k_{t}\right)$, as a function of the parameters of the plasma, of the mass velocities of the uniform regions and of the wavenumber $\mathbf{k}_{t} \equiv\left(k_{x}, 0, k_{z}\right)$ of the perturbation of the interface. Then an algebraic dispersion relation is obtained, whose roots yield $v$.

Within the incompressible MHD approximation (IMHD) it is possible to derive a simple formula for the phase velocity. Because of this, the IMHD result is often employed in space physics to interpret the observed data and to examine the stability of the configuration. Sometimes the tacit assumption is made that the compressibility effects thus neglected should (if any) improve stability. This assumption is based on well known theorems (see for example [?]) derived from a variational principle, according to which compressibility leads to a positive contribution to the energy of the plasma and so tends to stabilize it. However, these results cannot be applied here, since due to the presence of mass flow the plasma can sustain perturbations with a negative energy density that can lead to instability. This is a consequence of the transformation properties of the energy density $W$ of a perturbation of wavenumber $\mathbf{k}_{t}$ and frequency $\omega$ under Galilean transformations. Let the primes denote the quantities calculated in the reference frame of the plasma that moves with a velocity $\mathbf{u} \equiv\left(u_{x}, 0, u_{z}\right)$ with respect to the observer, and the quantities without primes refer to the observer frame. Then we have $W^{\prime} / \omega^{\prime}=W / \omega$. Notice that the Dopplershifted frequency in the plasma frame $\omega^{\prime}=\omega-\mathbf{u} \cdot \mathbf{k}_{t}$ can be negative if $u_{k}=\mathbf{u} \cdot \mathbf{k}_{t} / k_{t}$ is sufficiently large (the phase velocity in the plasma frame is then $v^{\prime}=v-u_{k}$ ). Since $W^{\prime}$ is always positive, the energy density of the perturbation as measured in the laboratory frame is then negative. A negative energy density perturbation may lead to instability if it couples to a positive energy density perturbation, since both can grow without an external source of energy.

In IMHD, the instability arises from perturbations of the interface that are exponentially damped in both plasma regions (like surface gravity waves in water). The penetration depth of these perturbations is equal to their wavelength $2 \pi / k_{t}$. The instability occurs when $u_{k}$ exceeds a critical value $u_{i}$ (see below), such that their energy density (in the laboratory frame) is positive in one region and negative in the other. These perturbations grow because energy is transferred across the interface from the negative energy density region to the other, and remains localized as it can not be transported away across the magnetic field. This instability will be called primary KHI. Notice that the Alfvén wave only transports energy along the field lines, and does not participate in the KHI if the transition region has a vanishing thickness.

The essential difference between IMHD and compressible MHD (CMHD) is that in the latter there are perturbations (the fast and slow magnetosonic waves) that propagate in the bulk of the plasma and transport energy across the magnetic field (see for example [?]) Consequently, the effect of compressibility on the Kelvin-Helmholtz instability is complex, leading to the stabilization of certain perturbations, the destabilization of others, and the occurrence of modes that have no analogue in IMHD and that may lead to new instabilities. Let us briefly discuss the basic physics involved.

Consider first the primary KHI. Due to compressibility, if $u_{k}$ is sufficiently large the perturbation may be able to propagate in both regions as fast magnetosonic waves. When this happens, the perturbation is stable, and it is seen by the observer as a pair of fast magnetosonic waves radiated away from the interface. One of these waves has a negative, and the other a positive energy density [?], [?]. In this way, perturbations that are unstable according to IMHD are stabilized by compressibility. However, in addition to this large- $u_{k}$ stabilization, there is another effect of compressibility on the primary KHI, namely that the critical value $u_{c}$ 
for the onset of the instability is lowered $\left(u_{c} \leq u_{i}\right)$. This is a destabilizing effect, since perturbations that are stable according to IMHD become unstable when compressibility is taken into account. As we shall show later, this effect is important in the magnetopause. The penetration depth of the unstable modes is also affected by compressibility, and is no longer given by $2 \pi / k_{t}$.

Yet, there are further effects of compressibility. Due to the existence of the slow magnetosonic waves new kinds of perturbations of the interface are possible, that have no counterpart in IMHD. Some of these perturbations are stable evanescent oscillations, and other are slow magnetosonic waves in both regions (radiation of a pair of slow magnetosonic waves). However, some of these new perturbations may be unstable. These new instabilities (called secondary KHI) are found in intervals of $u_{k}$ that correspond to stable perturbations according to IMHD. They lie totally, or partially, below the critical value $u_{c}$ for the onset of the primary KHI. The occurrence of the secondary KHI is an additional destabilizing effect of compressibility. The growth rate of the secondary modes is usually small. However, we shall see that these modes cannot be ignored, since in some configurations of the magnetopause they are the only unstable modes present.

A substantial contribution to the understanding of the compressibility effects on the Kelvin-Helmholtz instability was given by Miura and Pritchett [?]. However, these effects have not yet been fully explored for general configurations with density and temperature discontinuities as well as magnetic field shear, such as those occurring in the magnetopause. For an extensive revision of the literature and of the main results on this subject, see refs. [?], [?]. Recently, we have developed techniques [?], [?], [?] to analyze the stability of an interface separating two uniform flows, with arbitrary jumps in the velocity, magnetic field, density and temperature, and with no restrictions on the wave vector of the perturbation.

The content of this paper is as follows. In Section II we outline the basic theory. In Section III we explain the techniques for analyzing the dispersion relation and the characteristics of the unstable modes. Section IV discusses the secondary KHI. A useful graphical technique that simplifies the complexity of the stability analysis is given in Section V. The physical parameters used in the applications to the magnetopause stability are introduced in Section VI. The results for two examples, (i) a model of the front side magnetopause, and (ii) an event observed at the near equatorial flank of the magnetopause are given in Section VII. We determine the stability of these configurations, and study the unstable modes that may occur in them. The final remarks are in Section VIII. We conclude that the effects of compressibility may introduce important changes with respect to the predictions of IMHD.

\section{Theory}

We consider two semiinfinite, uniform plasma regions in relative motion. The geometry of the problem is shown in Fig. 1. Region $1(y>0)$, moves with a constant velocity $\mathbf{u}_{1}^{\prime}$, and region $2(y<0)$ moves with a constant velocity $\mathbf{u}_{2}^{\prime}$. The velocities $\mathbf{u}_{1}^{\prime}$ and $\mathbf{u}_{2}^{\prime}$ and the magnetic fields $\mathbf{B}_{1}$ and $\mathbf{B}_{2}$ are parallel to the interface (suffixes $i=1,2$ denote quantities pertaining to region 1 and 2, respectively). At the interface, $p, \rho$, and $\mathbf{B}$ can have arbitrary discontinuities, subject to the equilibrium condition

$$
p_{1}+\frac{B_{1}^{2}}{8 \pi}=p_{2}+\frac{B_{2}^{2}}{8 \pi}
$$
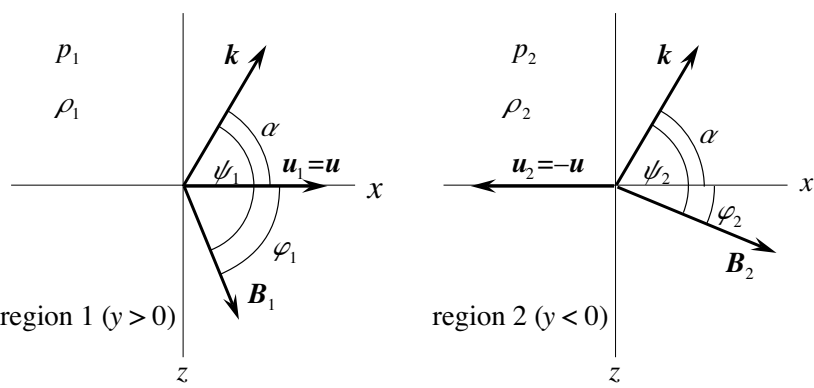

Figure 1. Geometry of the problem.

We shall assume ideal CMHD and consider the linear adiabatic perturbations of this configuration. Since the growth rate of the instability does not depend on $\mathbf{u}_{1}^{\prime}$ and $\mathbf{u}_{2}^{\prime}$ separately, but only on their difference $2 \mathbf{u}=\mathbf{u}_{1}^{\prime}-\mathbf{u}_{2}^{\prime}$, we use a reference frame moving with the average velocity $\mathbf{u}^{\prime}=\left(\mathbf{u}_{1}^{\prime}+\mathbf{u}_{2}^{\prime}\right) / 2$. In this frame $\mathbf{u}_{1}=\mathbf{u}=u \mathbf{e}_{x}, \mathbf{u}_{2}=-\mathbf{u}$, and $\alpha$ is the angle between $\mathbf{u}$ and the wavenumber $\mathbf{k}_{t} \equiv\left(k_{x}, 0, k_{z}\right)$ of a perturbation whose frequency is $\omega$; the phase velocity is then $v=\omega / k_{t}$. Once the phase velocity of the perturbation is found, we can obtain its value in the observer frame by means of the Galilean transformation $v^{\prime}=v-u_{k}^{\prime}$.

The (Doppler-shifted) frequencies in the plasma frames are $\omega_{1}=\omega-u_{k} k_{t}$ in region 1 and $\omega_{2}=\omega+u_{k} k_{t}$ in region 2 , and the corresponding phase velocities are

$$
v_{1}=v-u_{k} \quad, \quad v_{2}=v+u_{k}
$$

We shall denote by $\psi_{i}$ the angles between $\mathbf{B}_{i}$ and $\mathbf{k}_{t}$, and by $\varphi_{i}$ the angles between $\mathbf{B}_{i}$ and $\mathbf{u}$. The angle between $\mathbf{k}_{t}$ and $\mathbf{u}$ is $\alpha$, so that $u_{k}=u \cos \alpha$. We employ the following notation: 


$$
\begin{gathered}
A_{i}=\frac{B_{i}}{\sqrt{4 \pi \rho_{i}}}=\text { Alfvén velocity }, \quad S_{i}=\sqrt{\frac{\gamma p_{i}}{\rho_{i}}}=\operatorname{Sound} \operatorname{velocity}(\gamma=5 / 3) \\
d_{i}=\sqrt{A_{i}^{2}+S_{i}^{2}} \quad, \quad a_{i}=A_{i} \cos \psi_{i}, \quad b_{i}=\left(A_{i} S_{i} / d_{i}\right) \cos \psi_{i} \\
q_{i}=\sqrt{\frac{1}{2}\left(d_{i}^{2}+\sqrt{d_{i}^{4}-4 d_{i}^{2} b_{i}^{2}}\right.}, \quad m_{i}=\sqrt{\frac{1}{2}\left(d_{i}^{2}-\sqrt{d_{i}^{4}-4 d_{i}^{2} b_{i}^{2}}\right.} \\
\Gamma_{i}=\sqrt{-\frac{\left(v_{i}^{2}-q_{i}^{2}\right)\left(v_{i}^{2}-m_{i}^{2}\right)}{d_{i}^{2}\left(v_{i}^{2}-b_{i}^{2}\right)}}, \quad \text { with } \operatorname{Re}\left(\Gamma_{i}\right)>0, \text { or } \operatorname{Im}\left(\Gamma_{i}\right)>0 \text { if } \operatorname{Re}\left(\Gamma_{i}\right)=0
\end{gathered}
$$

In each one of the uniform regions, the general form of the perturbation is

$$
\zeta_{i}=e^{i\left(k_{t} x-\omega t\right)}\left(C_{i} e^{-k_{t} \Gamma_{i} y}+D_{i} e^{k_{t} \Gamma_{i} y}\right)
$$

In this formula, $\zeta_{i}$ denotes the $y$-component of the displacement, and $C_{i}$ and $D_{i}$ are constants. The penetration depth of the perturbation in region $i$ is proportional to $1 / \operatorname{Re}\left(\Gamma_{i}\right)$.

When $\operatorname{Re}\left(\Gamma_{i}\right)=0$, it can be recognized that eq. (6) is the dispersion relation for the fast and slow magnetosonic waves in a semiinfinite uniform moving plasma; their wave vector is $\left(k_{x}, k_{t} \operatorname{Im}\left(\Gamma_{i}\right), k_{z}\right)$. When $\operatorname{Re}\left(\Gamma_{i}\right) \neq 0$, eq. (6) is the dispersion relation for evanes-

$$
\mathrm{T}=\frac{\rho_{1}\left(v_{1}^{2}-a_{1}^{2}\right)}{\Gamma_{1}}-\frac{\rho_{2}\left(v_{2}^{2}-a_{2}^{2}\right)}{\Gamma_{2}} \quad, \quad \mathrm{~S}=\frac{\rho_{1}\left(v_{1}^{2}-a_{1}^{2}\right)}{\Gamma_{1}}+\frac{\rho_{2}\left(v_{2}^{2}-a_{2}^{2}\right)}{\Gamma_{2}}
$$

The matrix equation (8) may be used to investigate many problems involving localized modes as well as the reflection and transmission of magnetosonic waves. Since unstable modes and stable surface oscillations have $\operatorname{Re}\left(\Gamma_{1,2}\right) \neq 0$, we must have $C_{1}=D_{2}=0$ and then the corresponding dispersion relation is

$$
\mathrm{T}=\frac{\rho_{1}\left(v_{1}^{2}-a_{1}^{2}\right)}{\Gamma_{1}}-\frac{\rho_{2}\left(v_{2}^{2}-a_{2}^{2}\right)}{\Gamma_{2}}=0
$$

The propagating modes have $\operatorname{Re}\left(\Gamma_{1,2}\right)=0$, so that there is no restriction on the $C_{i}, D_{i}$. In this case, the conditions $\mathrm{T}=0$ and $\mathrm{S}=0$ determine the poles and the zeros of the reflection coefficient. Notice that the sign of the $y$-component of the group velocity of magnetosonic cent perturbations, and of course in this case the amplitudes of the exponentially growing perturbations $\left(D_{1}\right.$ and $C_{2}$ ) in eq. (7) must vanish since the plasma extends to $(+$ or -$)$ infinity.

The boundary conditions at $y=0$ (continuity of $\zeta$ and of the normal stress) couple the perturbations of regions 1 and 2. The coupling leads to a matrix equation that relates the amplitudes $C_{i}, D_{i}$ :

$$
\left[\begin{array}{c}
C_{1} \\
D_{1}
\end{array}\right]=\frac{\Gamma_{1}}{2 \rho_{1}\left(v_{1}^{2}-a_{1}^{2}\right)}\left[\begin{array}{cc}
\mathrm{T} & \mathrm{S} \\
\mathrm{S} & \mathrm{T}
\end{array}\right]\left[\begin{array}{c}
C_{2} \\
D_{2}
\end{array}\right]
$$

Here

waves may be different from the sign of $\operatorname{Re}\left(\Gamma_{1,2}\right)$. Then one must examine the group velocities in each region, to ascertain which of these conditions yields the dispersion relation for radiation of a pair of waves, or for the total transmission of waves (and their time-reversed processes).

\section{Classification of the modes}

To investigate the roots of the dispersion relation (10) it is useful to introduce a polynomial $P$ associated to it. It is obtained by taking the numerator of the product TS, thus eliminating the square roots:

$$
P=\rho_{1}^{2} d_{1}^{2}\left(v_{1}^{2}-a_{1}^{2}\right)^{2}\left(v_{1}^{2}-b_{1}^{2}\right)\left(v_{1}^{2}-q_{1}^{2}\right)\left(v_{1}^{2}-m_{1}^{2}\right)-\rho_{2}^{2} d_{2}^{2}\left(v_{2}^{2}-a_{2}^{2}\right)^{2}\left(v_{2}^{2}-b_{2}^{2}\right)\left(v_{2}^{2}-q_{2}^{2}\right)\left(v_{2}^{2}-m_{2}^{2}\right)
$$


Clearly, the roots of $P=0$ encompass the solutions of $\mathrm{T}=0$ ('true' roots) as well as those of $\mathrm{S}=0$ ('spurious' roots), and we must discard the latter when we look for localized modes. Accordingly, we call 'true branch' $(\mathrm{T})$ the set of solutions of $\mathrm{T}=0$, and 'spurious branch' ( $\mathrm{S}$ ) the solutions of $\mathrm{S}=0$. The $\mathrm{T}$ branch and the $\mathrm{S}$ branch consist of several pieces (that we call 'segments'), each of which is a continuous manifold in the parameter space of the problem. They are arranged in such a way that any given $\mathrm{T}$ segment is bounded by one or more S segments (and vice-versa). Let us consider a root $v$ (real or complex) of $\mathrm{P}$ that belongs to a certain segment $\mathrm{T}_{j}$. If the parameters change, $v$ moves along $\mathrm{T}_{j}$ until eventually it arrives to the boundary between $\mathrm{T}_{j}$ and a segment $\mathrm{S}_{l}$ of $\mathrm{S}$. As it crosses this boundary, $v$ ceases to belong to the true branch and becomes spurious. It is important to notice that the T-S boundaries occur only for $v$ real, at the points where $\Gamma_{1,2}=0, \infty$. This is fortunate, since it simplifies considerably the analysis of the topology of the $\mathrm{T}$ and $\mathrm{S}$ branches.

A convenient way to visualize the real roots of the dispersion relation (10) and so unravel the topology of the $\mathrm{T}$ and $\mathrm{S}$ branches is to use a graphical method introduced by Chandrasekhar [?]. This method takes advantage of the fact that the problem of finding the roots $v$ of $P=0$ is equivalent to solve for $v_{1}$ and $v_{2}$ the coupled equations

$$
\begin{aligned}
& P\left(v_{1}, v_{2}\right)=0 \\
& v_{2}=v_{1}+2 u_{k}
\end{aligned}
$$

From any given solution $\left(v_{1}, v_{2}\right)$ of the system (12), one can obtain a root $v=\left(v_{1}+v_{2}\right) / 2$ of $P=0$. The real solutions of this system can be easily found graphically in a $\left(v_{1}, v_{2}\right)$ diagram, as the intersections of the line $\mathrm{L}$ given by $(12 b)$ with the curves that are obtained solving the bicubic (in $v_{1}$ and $v_{2}$ ) equation (12a) for $v_{2}$.

In Fig. 2 we show a typical Chandrasekhar diagram, corresponding to the plasma configuration (d) investigated in Section VII (notice that the scale of $v_{2}$ is different from that of $v_{1}$ for convenience in drawing). It suffices to consider a single quadrant, since the curves $v_{2}=v_{2}\left(v_{1}\right)$ are symmetrical under reflections on both axes. In Fig. 2, the lines $v_{2}=v_{1}$ and $v_{2}=-v_{1}$ represent the $v$ - and $u_{k}$ - axes (except from a scale factor $\sqrt{2})$.

The perturbation in the region $i(i=1,2)$ is a slow magnetosonic wave if $b_{i} \leq\left|v_{i}\right| \leq m_{i}$, and a fast magnetosonic wave if $q_{i} \leq\left|v_{i}\right|$. It is evanescent if $0 \leq\left|v_{i}\right| \leq b_{i}$ or $m_{i} \leq\left|v_{i}\right| \leq q_{i}$ (we shall call the latter the 'slow' and 'fast' evanescent perturbations, not to be confused with the slow and fast magnetosonic waves). A real root belongs to the $\mathrm{T}$ branch if $\operatorname{Sign}\left(v_{1}^{2}-a_{1}^{2}\right) \neq \operatorname{Sign}\left(v_{2}^{2}-a_{2}^{2}\right)$ (i. e., if $\left|v_{1}\right|<a_{1}$ and $\left|v_{2}\right|>a_{2}$, or $\left|v_{1}\right|>a_{1}$ and $\left.\left|v_{2}\right|<a_{2}\right)$; otherwise, it belongs to the $\mathrm{S}$ branch.

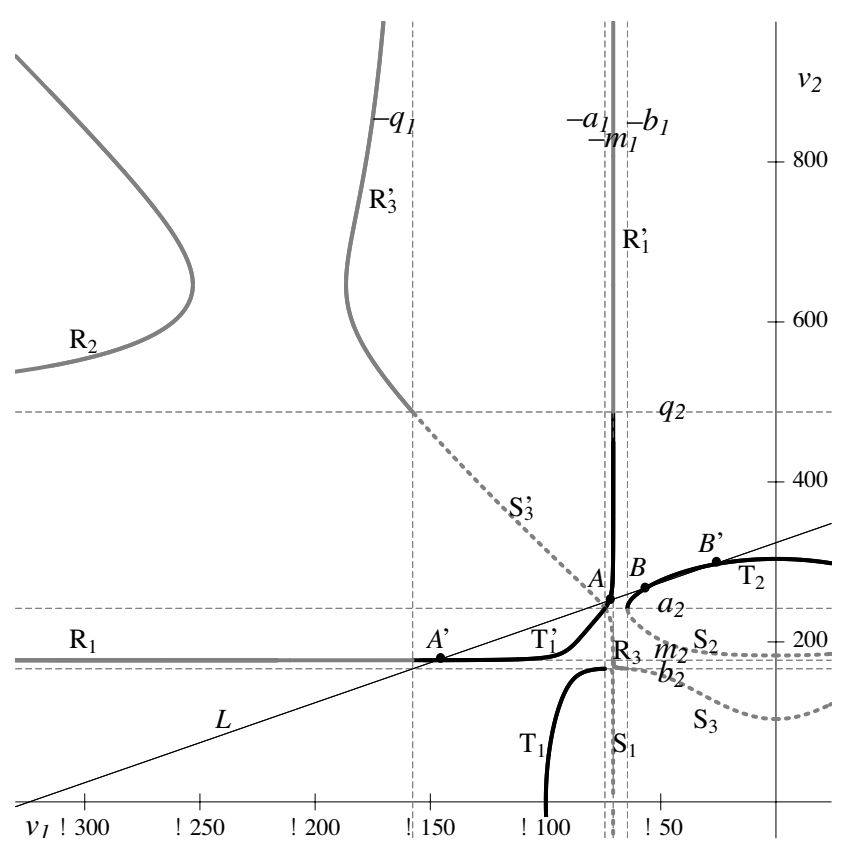

Figure 2. Chandrasekhar diagram for the configuration of case (d) (Section VII) and $\psi_{1}=150^{\circ}$. The fine dashed lines labeled (left to right) $-q 1,-a 1,-m 1,-b 1$ and $q 2$, $a 2, m 2, b 2$ (top to bottom) mark the boundaries where the perturbation changes its character.

Both the $\mathrm{T}$ and $\mathrm{S}$ branches must be considered for the $y$-propagating modes (radiated or totally transmitted magnetosonic waves). It can be shown that all the roots of this type in the second and fourth quadrants of the $\left(v_{1}, v_{2}\right)$ diagram correspond to radiation and those in the first and third quadrant to total transmission. According to these criteria we can classify the branch segments shown in Fig. 2. The true evanescent segments are represented by solid curves (labeled $\mathrm{T}_{1}, \mathrm{~T}_{1}^{\prime}$, $\mathrm{T}_{2}$ ), the spurious evanescent segments by gray dotted curves (labeled $\mathrm{S}_{1}, \mathrm{~S}_{2}, \mathrm{~S}_{3}, \mathrm{~S}_{3}^{\prime}$ ), and the radiation segments by gray solid lines (labeled $\mathrm{R}_{1}, \mathrm{R}_{1}^{\prime}, \mathrm{R}_{2}, \mathrm{R}_{3}, \mathrm{R}_{3}^{\prime}$ ).

For any given $u_{k}$ there are five pairs of roots of $P(v)=0$. Two pairs are always real, and are uninteresting for us since they are always spurious, or correspond to totally transmitted modes (they do not appear in Fig. 2 since they lie in the first and third quadrants). One root of a third pair lies on the curve formed by the segments labeled with the suffix 3 in Fig. $2\left(\mathrm{~S}_{3}, \mathrm{R}_{3}\right.$, $\mathrm{S}_{3}^{\prime}, \mathrm{R}_{3}^{\prime}$ ), or on its continuation in the first quadrant. The other root of this pair is found on a symmetrical curve lying in the third and fourth quadrants. This pair of roots is also uninteresting, since they are always either spurious or propagating modes. The remaining two pairs of roots, labeled $A, A^{\prime}$ and $B, B^{\prime}$, are important to us since they can be unstable. For the value of $u_{k}$ represented in Fig. 2 all these roots are real and represent stable surface oscillations, since the points lie on the segments $T_{1}^{\prime}$ and $T_{2}$, respectively.

If we consider a larger value of $u_{k}$, the line $\mathrm{L}$ must be displaced upwards, parallel to itself. Then the points $B, B^{\prime}$ move on the segment $\mathrm{T}_{2}$ until they coalesce for 
$u_{k}=u_{d 2}$ when $L$ is tangent to $\mathrm{T}_{2}$. For $u_{k}>u_{d 2}$ the roots $v_{B}, v_{B^{\prime}}$ are complex conjugate, but they still belong to the $\mathrm{T}$ branch (since roots can pass to the other branch only for $v$ real). Then $u_{k}=u_{d 2}$ corresponds to marginal stability, and one of the roots $v_{B}, v_{B^{\prime}}$ is unstable for $u_{k}>u_{d 2}$ (the other is damped). This instability persists until $u_{k}=u_{d 6}$, when $\mathrm{L}$ is tangent to the segment $R_{2}$. Then $u_{d 6}$ corresponds also to marginal stability, and for $u_{k}>u_{d 6}, v_{B}, v_{B^{\prime}}$ are stable radiation modes. On the other hand, if one considers values of $u_{k}$ larger than that represented in Fig. 2, the points $A, A^{\prime}$ move along $\mathrm{T}_{1}^{\prime}$ and eventually pass into the segments $\mathrm{R}_{1}, \mathrm{R}_{1}^{\prime}$, and then $v_{A}, v_{A^{\prime}}$ correspond to radiation modes.

Let us now consider smaller values of $u_{k}$. As $u_{k}$ is reduced, L moves downwards. The points $B, B^{\prime}$ move on $\mathrm{T}_{2}$ until they pass into $\mathrm{S}_{2}$. It can be shown that for $u_{k}>0$, no new instability arises from the roots $v_{B}, v_{B^{\prime}}$. On the other hand, the points $A, A^{\prime}$ move along $\mathrm{T}_{1}^{\prime}$ until they coalesce for $u_{k}=u_{d 3}$. When this happens, the double root $v_{A}=v_{A^{\prime}}$ is marginally stable. For $u_{d 1}<u_{k}<u_{d 3}$ we have instability, and $v_{A}, v_{A^{\prime}}$, are complex conjugate. Marginal stability is again achieved at $u_{k}=u_{d 1}$. For $u_{k}<u_{d 1}$ the points $A, A^{\prime}$ move along $\mathrm{T}_{1}$ until they pass into $\mathrm{S}_{1}$. No other instability related to $v_{A}, v_{A^{\prime}}$ occurs for $u_{k}>0$. Using diagrams like Fig. 2 it is possible to investigate the stability of any configuration of interest. However, this method is unpractical, since we need a new diagram for each orientation of $\mathbf{k}_{t}$, and in addition, we cannot determine the growth rate of the unstable modes.

In the case shown in Fig. 2 there are two unstable intervals (for $u_{k}>0$ ). The first (in order of increasing $u_{k}$ ) involves $v_{A}, v_{A^{\prime}}$ and occurs for $u_{d 1}<u_{k}<u_{d 3}$. The second occurs for $u_{d 2}<u_{k}<u_{d 6}$, and involves $v_{B}, v_{B^{\prime}}$. This result is due to the special choice of parameters of Fig. 2. In general, there can be up to six positive values of $u_{k}$ corresponding to significant (i.e., belonging to the $\mathrm{T}$ branch) double roots of $P(v)=0$, that we label $u_{d 1}, \ldots, u_{d 6}$. Correspondingly, there can be up to three unstable intervals, namely

$$
\begin{aligned}
& u_{d 1}<u_{k}<u_{d 3} \text { secondary interval A } \\
& u_{d 2}<u_{k}<u_{d 4} \text { secondary interval B }
\end{aligned}
$$

$$
u_{d 5}<u_{k}<u_{d 6} \quad \text { primary interval }
$$

As the parameters are varied, it may happen that the pair $\left(u_{d 3}, u_{d 5}\right)$, or the pair $\left(u_{d 4}, u_{d 5}\right)$, coalesces and becomes complex. In the first case, the secondary interval $A$ merges with the primary interval. In the second case, the secondary interval B merges with the primary interval (as in Fig. 2). In addition the secondary intervals $(13 \mathrm{a}, \mathrm{b})$ may also overlap partially or totally. The secondary intervals can be extremely narrow for some values of the parameters, in particular they vanish when the perturbations are flutes in either region 1 or 2. For negative $u_{k}$, a completely symmetrical result is obtained, in which the signs of $u_{d 1}, \ldots, u_{d 6}$ are changed, the inequalities (13a-c) are reversed, and the roles of the root pairs $\left(A, A^{\prime}\right)$ and $\left(B, B^{\prime}\right)$ are exchanged. There are no simple formulae for $u_{d 1}, \ldots, u_{d 6}$.

To find the double roots $v_{d 1}, \ldots, v_{d 6}$ of $P(v)$ and the corresponding $u_{k}$ values $u_{d 1}, \ldots, u_{d 6}$ we must solve numerically for $(u, v)$ the system

$$
P(u, v)=0 \quad, \quad \partial P(u, v) / \partial v=0
$$

and discard the roots belonging to the $\mathrm{S}$ branch. The unstable intervals can then be mapped in the parameter space of the configuration (see Section V).

\section{The secondary Kelvin- Helmholtz instability}

With the help of diagrams like Fig. 2 it is easy to understand the role of the compressibility on the KHI. As $S_{i}$ increases, $b_{i}$ and $m_{i}$ approach $a_{i}$, and $q_{i}$ increases without bound, so that in the incompressible limit $\left(S_{1}, S_{2} \rightarrow \infty\right)$ the secondary unstable intervals disappear, and we are left only with the primary unstable interval, that now extends to infinity. The secondary KHI is then a consequence of compressibility, as well as the large $u_{k}$ stabilization of the primary KHI at $u_{d 6}$. It is enlightening to see in more detail the origin of the secondary KHI. Notice that $P\left(v_{1}, v_{2}\right)$ can be written in the form

$$
P\left(v_{1}, v_{2}\right)=S_{1}^{2} S_{2}^{2} P_{i n c}\left(v_{1}, v_{2}\right)+S_{1}^{2} P_{1}\left(v_{1}, v_{2}\right)+S_{2}^{2} P_{2}\left(v_{1}, v_{2}\right)+P_{3}\left(v_{1}, v_{2}\right)
$$

In $(15)$

$$
P_{\text {inc }}\left(v_{1}, v_{2}\right)=\left(v_{1}^{2}-a_{1}^{2}\right)\left(v_{2}^{2}-a_{2}^{2}\right)\left[\rho_{1}^{2}\left(v_{1}^{2}-a_{1}^{2}\right)^{2}-\rho_{2}^{2}\left(v_{2}^{2}-a_{2}^{2}\right)^{2}\right]
$$


and $P_{1}, P_{2}, P_{3}$, are polynomials whose coefficients are functions of $a_{1}, a_{2}, A_{1}, A_{2}, \rho_{1}$ and $\rho_{2}$. Then, in the limit $S_{1}, S_{2} \rightarrow \infty$, if we assume that $v_{1,2}$ remain finite, eq. (12a) reduces to $P_{i n c}=0$, whose solutions are:

$$
\begin{aligned}
& v_{1}= \pm a_{1} \\
& v_{2}= \pm a_{2}
\end{aligned}
$$

$$
\begin{aligned}
& v_{2}= \pm \sqrt{a_{2}^{2}-\frac{\rho_{1}}{\rho_{2}}\left(v_{12}^{2}-a_{1}^{2}\right)} \\
& v_{2}= \pm \sqrt{a_{2}^{2}+\frac{\rho_{1}}{\rho_{2}}\left(v_{12}^{2}-a_{1}^{2}\right)}
\end{aligned}
$$

These solutions yield, respectively, the roots

$$
\begin{gathered}
v=v_{a 1 \pm}=u_{k} \pm a_{1} \\
v=v_{a 2 \pm}=-u_{k} \pm a_{2} \\
v=v_{K \pm}=u_{k} \frac{\rho_{1}-\rho_{2}}{\rho_{1}+\rho_{2}} \pm \frac{\sqrt{\left(a_{1}^{2} \rho_{1}+a_{2}^{2} \rho_{2}\right)\left(\rho_{1}+\rho_{2}\right)-4 u_{k}^{2} \rho_{1} \rho_{2}}}{\rho_{1}+\rho_{2}} \\
v=v_{S \pm}=u_{k} \frac{\rho_{1}+\rho_{2}}{\rho_{1}-\rho_{2}} \pm \frac{\sqrt{\left(a_{1}^{2} \rho_{1}-a_{2}^{2} \rho_{2}\right)\left(\rho_{1}-\rho_{2}\right)+4 u_{k}^{2} \rho_{1} \rho_{2}}}{\rho_{1}-\rho_{2}}
\end{gathered}
$$

The roots (17a) and (17b) are stable, and correspond to Alfvén waves propagating in regions 1 and 2, respectively. The roots $(17 c)$ have been discussed by Axford [?],[?], Chandrasekhar [?] and Southwood [?]. They yield the incompressible (primary) Kelvin-Helmholtz instability for

$$
\left|u_{k}\right|>u_{i}=\frac{1}{2} \sqrt{\frac{\rho_{1} \rho_{2}}{\rho_{1}-\rho_{2}}\left(a_{1}^{2} \rho_{1}+a_{2}^{2} \rho_{2}\right)}
$$

Finally, the roots $(17 d)$ are spurious.

Some of the roots $(17 a-d)$ can coincide for special values of $u_{k}$. For example, when

$$
u_{k}=u_{D}=\frac{1}{2}\left(a_{1}+a_{2}\right)
$$

the roots $v_{a 1-}, v_{a 2+}, v_{K \pm}(-$ or + according to the sign of $\left.a_{1}^{2} \rho_{1}-a_{2}^{2} \rho_{2}\right)$ and $v_{S-}$ coincide and their value is

$$
v=v_{D}=\frac{1}{2}\left(-a_{1}+a_{2}\right)
$$

This degeneracy corresponds to the point $\mathrm{D}_{1}\left(v_{1}=\right.$ $\left.-a_{1}, v_{2}=-a_{2}\right)$, where the lines $(16 a-d)$ cross. It can be verified that $u_{i} \geq u_{D}$, and that the equality holds only for $a_{1}^{2} \rho_{1}-a_{2}^{2} \rho_{2}=0$. Let us now assume that $S_{1}, S_{2}$ are large, but finite. Then the term $S_{1}^{2} P_{1}\left(v_{1}, v_{2}\right)+S_{2}^{2} P_{2}\left(v_{1}, v_{2}\right)$ in $(15)$ can be treated as a small perturbation that couples the degenerate roots $v_{a 1-}, v_{a 2+}, v_{K+}\left(\right.$ or $\left.v_{K-}\right), v_{S_{-}}$. It can be shown that part of the degeneracy is removed, and some roots acquire an imaginary part. The unstable modes so arising (secondary KHI) have no analogue among the purely incompressible modes. It is interesting to observe that this instability appears around $u_{k} \approx u_{D}$, that is, in a range of $u_{k}$ that is stable according to IMHD, since it lies below $u_{i}$.

In addition to $\mathrm{D}_{1}$, there are three other points where degeneracy of the roots $(17 a-d)$ occurs, namely $\mathrm{D}_{2}$ $\left(u=-u_{D}, v=-v_{D}\right), \mathrm{D}_{3}\left(u=v_{D}, v=u_{D}\right)$ and $\mathrm{D}_{4}$ $\left(u=-v_{D}, v=-u_{D}\right)$. The removal of the degeneracy at $\mathrm{D}_{2}$ due to compressibility leads to overstable modes like at $\mathrm{D}_{1}$. On the other hand, the removal of the degeneracies at $D_{3}$ and $D_{4}$ does not lead to instabilities.

\section{Stability diagrams}

The problem of finding the localized eigenmodes is straightforward, if tedious, since we must calculate numerically the roots of the polynomial $P$ (of degree ten), that depends on seven independent parameters. Six of them characterize the plasma configuration; they can be taken as the ratios $r_{b}=B_{2} / B_{1}, r_{s}=S_{2} / S_{1}$ and $r_{d}=\rho_{2} / \rho_{1}$, the magnitude $u$ of the relative velocity, and the angles $\varphi_{1}$ from $\mathbf{B}_{1}$ to $\mathbf{u}$, and $\theta$ from $\mathbf{B}_{1}$ to $\mathbf{B}_{2}$ (magnetic shear angle). The remaining is the angle $\psi_{1}$ from $\mathbf{B}_{1}$ to $\mathbf{k}_{t}$, and identifies the perturbation we are considering.

Notice, however, that $P$ (and its roots) depends on $u$ only through the combination

$$
u_{k}=u \cos \alpha=u \cos \left(\psi_{1}-\varphi_{1}\right)
$$


Accordingly, we shall represent the results of the analysis by means of diagrams in which we plot the growth rate $\operatorname{Im}(v)$ of the unstable modes as functions of $\psi_{1}$ and $u_{k}$, keeping fixed the remaining parameters $\left(r_{b}, r_{s}\right.$, $\left.r_{d}, \theta\right)$ that determine $P$. An example is the stability diagram shown in Fig. 3, in which we have drawn a contour plot of $\operatorname{Im}(v)$ for the parameters of a case investigated in Section VI (case $(\mathrm{d}), r_{b}=1.5, r_{s}=2.67$, $r_{d}=0.117, \theta=-80^{\circ}$ ). In Fig. 3 we have also drawn the curve $u=u_{i}\left(\psi_{1}\right)$ that gives the marginal stability condition (18) according to the incompressible model. All the points of the stability diagram above $u_{i}\left(\psi_{1}\right)$ correspond to instability according to IMHD. Notice that the contour plot does not refer to a unique plasma configuration, since we have not yet specified $u$ and $\varphi_{1}$. Then it can be used for all the configurations having the same values of $r_{b}, r_{s}, r_{d}$ and $\theta$. This is a distinct advantage since with a single graph we can study the effects of changing the magnitude of the relative velocity and its orientation with respect to the magnetic field.

In Fig. 3, we can see the main effects of compressibility: the large- $u_{k}$ stabilization and the small- $u_{k}$ destabilization of the primary instability, and the presence of the secondary instability in $u_{k}$ intervals that are stable according to IMHD. It should also be mentioned that for $u_{k}$ appreciably larger than $u_{i}\left(\psi_{1}\right)$ (i. e., not very close to it) the growth rates calculated with CMHD are always smaller than those calculated with IMHD (17c).

To investigate a particular configuration by means of the stability diagram we must draw the curve $\mathrm{C}$ given by eq. (21), that depends on the two remaining parameters $\left(u, \varphi_{1}\right)$ that characterize it. Following $\mathrm{C}$ we find the value of $\operatorname{Im}(v)$ for this configuration, for any perturbation characterized by the angle $\psi_{1}$. Since the problem is invariant under the transformation $(v \rightarrow-v$, $u_{k} \rightarrow-u_{k}$ ), it suffices to calculate the contour plot for $u_{k} \geq 0$; of course, we must then draw the curve $\mathrm{C}^{\prime}$ given by $u_{k}=-u \cos \alpha$ in addition to $\mathrm{C}$.

In the example shown in Fig. 3 we see that the configuration described by $\mathrm{C}$ and $\mathrm{C}^{\prime}\left(u=1.7 A_{1}, \varphi_{1}=\right.$ $\left.-89.9^{\circ}\right)$ is stable according to IMHD, but is unstable if compressibility is taken into account. The unstable modes occur in the (approximate) range $40^{\circ} \leq \psi_{1} \leq$ $50^{\circ}$ and they belong to the secondary KHI. Their maximum growth rate is small $\left(\operatorname{Im}(v) \approx 0.04 A_{1}\right)$, but significant. It can be observed that other configurations with the same values of $r_{b}, r_{s}, r_{d}$ and $\theta$ can be more unstable. The extreme of $\operatorname{Im}(v)$ in Fig. 3 is $\approx 2.115 A_{1}$; it occurs for $\psi_{1} \approx 25^{\circ}$ and $u_{k} \approx 5 A_{1}$, a value that requires a very large $u$.

The meaning of the large- $u_{k}$ stabilization can be clarified by means of an example. Let us consider in Fig. 3 a hypothetical configuration with $u=10 A_{1}$ and $\varphi_{1}=-89.9^{\circ}$. The new curves $\mathrm{C}_{\mathrm{n}}$ and $\mathrm{C}_{\mathrm{n}}^{\prime}$ are similar to $\mathrm{C}$ and $\mathrm{C}^{\prime}$, only scaled by the factor $10 / 1.7$. Part of $\mathrm{C}_{\mathrm{n}}$ (for $45^{\circ} \leq \psi_{1} \leq 113^{\circ}$ ) lies in the upper part of the stability diagram $\left(u_{k}>u_{d 6}\right)$ corresponding to stable perturbations: these are the large- $u_{k}$ modes stabilized by compressibility. However, this configuration is unstable, since $C_{n}$ and $C_{n}^{\prime}$ pass through the unstable part of the diagram (actually, its most unstable modes have $\operatorname{Im}(v)$ very close to the extreme value for the diagram). Clearly, configurations having very large $u$ are always unstable, although large- $u_{k}$ stabilization may occur for some modes.

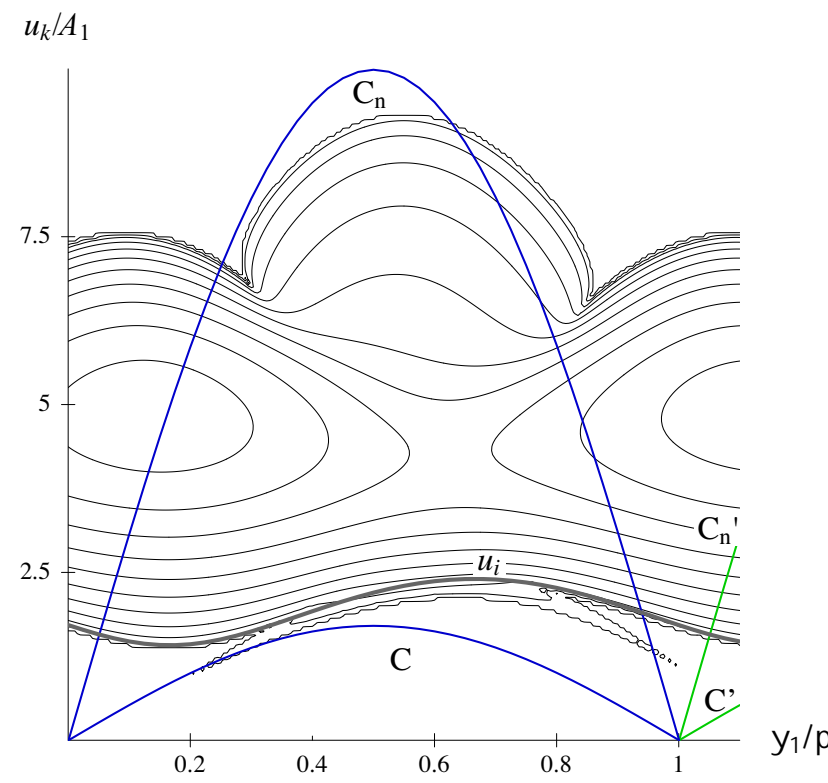

Figure 3. Stability diagram for case (d) of Section VII. The lines $\mathrm{C}$ and $\mathrm{C}^{\prime}$ correspond to the values of $u_{k}$ of the configuration, given by eq. (21). The contours of $\operatorname{Im}(v)$ are spaced by $0.2 A_{1}$. The large white areas at the top and bottom of the diagram correspond to stable perturbations.

A few comments must be made concerning stability diagrams like Fig. 3. To draw them we calculate the roots of the dispersion relation at discrete points of a grid in the $\left(\psi_{1}, u_{k}\right)$ plane. Sometimes, at the same point $\left(\psi_{1}, u_{k}\right)$ there are two unstable roots (this happens when the secondary intervals $\mathrm{A}$ and $\mathrm{B}$ overlap, or when any one of them overlaps parts of the primary interval). In these instances we show only the larger $\operatorname{Im}(v)$. The secondary intervals are very narrow in some $\left(\psi_{1}, u_{k}\right)$ regions, and may not show up, due to the coarseness of our grid (for the same cause some contours appear wavy in Fig. 3). Nothwithstanding these drawbacks, stability diagrams are extremely useful tools, since they provide an economical and fast way for finding the most interesting domains in parameter space. It is then possible to perform more precise and detailed calculations in these regions only, with confidence that we shall not miss anything relevant. 


\section{Parameters of magnetopause configurations}

Before discussing the application of the theory to the magnetopause, it is convenient to review how the parameters used in our formulae can be obtained from the theoretical models or from spacecraft data. We assume that the outside region (as seen from the Earth) is region 1 . In the applications, the parameters of the configuration (the flow velocities $\mathbf{u}_{1}^{\prime}$ and $\mathbf{u}_{2}^{\prime}$ and the angles $\psi_{B_{1}, u_{1}^{\prime}}$ and $\psi_{B_{1}, u_{2}^{\prime}}$ from $\mathbf{B}_{1}$ to $\mathbf{u}_{1}^{\prime}$ and $\mathbf{u}_{2}^{\prime}$, respectively) are given in a reference frame at rest with respect of the Earth. In our theory we use a reference frame moving with the average velocity $\mathbf{u}^{\prime}=\left(\mathbf{u}_{1}^{\prime}+\mathbf{u}_{2}^{\prime}\right) / 2$, then $\mathbf{u}_{1}=\mathbf{u}, \mathbf{u}_{2}=-\mathbf{u}$, with $\mathbf{u}=\left(\mathbf{u}_{1}^{\prime}-\mathbf{u}_{2}^{\prime}\right) / 2$. In what follows, all velocities are given in $\mathrm{km} / \mathrm{s}$. We employ the following formulae:

$$
\begin{gathered}
u=\frac{1}{2} \sqrt{u_{1}^{\prime 2}+u_{2}^{\prime 2}-2 u_{1}^{\prime} u_{2}^{\prime} \cos \theta_{u}}, \quad u^{\prime}=\frac{1}{2} \sqrt{u_{1}^{\prime 2}+u_{2}^{\prime 2}+2 u_{1}^{\prime} u_{2}^{\prime} \cos \theta_{u}}, \quad \theta_{u}=\psi_{B_{1}, u_{2}^{\prime}}-\psi_{B_{1}, u_{1}^{\prime}} \\
\varphi_{1}=\psi_{B_{1},-u_{1}^{\prime}}+\operatorname{Sign}\left(\sin \theta_{u}\right) \arccos \left(\frac{u_{1}^{\prime}-u_{2}^{\prime} \cos \theta_{u}}{2 u}\right)
\end{gathered}
$$

The angle $\delta$ from $\mathbf{u}$ to $\mathbf{u}^{\prime}$ is given by

$$
\delta=\operatorname{Sign}\left(\sin \theta_{u}\right) \arccos \left(\frac{u_{1}^{\prime 2}-u_{2}^{\prime 2}}{4 u u^{\prime}}\right)
$$

In eqs. (23) and (24) the factor $\operatorname{Sign}\left(\sin \theta_{u}\right)$ is needed to obtain the correct determination of $\varphi_{1}$ and $\delta$. Finally, the phase velocity of the perturbation in the Earth's frame is given by

$$
v^{\prime}=v-u^{\prime} \cos \left(\psi_{1}-\varphi_{1}-\delta\right)
$$

To consider the remaining parameters we must discuss separately the theoretical and spacecraft data.

\section{Data derived from theoretical models}

The conditions for the excitation of the KelvinHelmholtz instability on the dayside magnetopause were recently investigated in two papers. One, by Miura [?], reports numerical MHD simulations on the development of the instability, starting from hyperbolic tangent profiles of the unperturbed field quantities. One of the main results is that the magnetopause appears to be more KH unstable for northward IMF conditions than for southward pointing magnetic fields. However, the choice of parameters for the simulations is more adequate for the analysis of near flank configurations rather than dayside proper. The second paper [?] is based on a linear incompressible theory, and specifically addresses the distribution of the $\mathrm{KH}$ activity over the magnetopause surface. Charts of KHI growth rates, relying on input parameters provided by a MHD code that simulates the physical conditions of the frontside magnetosheath, are given. One of the main results is that under northward IMF conditions, the KH activity is restricted to some well defined regions of the magnetopause. Even when the IMF is due north, the activity decreases as the latitude increases from the magnetopause equator. In an third paper [?] the latitude dependence of the KHI is examined with a linear incompressible theory based on hyperbolic tangent profiles, using our incompressible theory for a tangential velocity discontinuity.

The data used here are given in Tables 1 and 2 . Those of Table 1 (also used in [?]) proceed from ref. [?] and were calculated with the magnetosheath model of ref. [?], which is also described in [?]. The IMF is assumed to point exactly north. The positions are at equator, middle latitude, and high latitude, along a meridian about 3 hours east of noon. The main difference among these positions is the increase of the local magnetic shear angle.

Table 1. Parameters of configurations of the front of the magnetopause according to the model.

\begin{tabular}{llcccccc}
\hline Case & Latitude & $f_{u^{\prime}}$ & $f_{b}$ & $f_{d}$ & $r_{b}$ & $\psi_{B_{1}, u_{1}^{\prime}}$ & $\theta$ \\
\hline (a) & low & 0.2352 & 1.1994 & 0.7502 & 1.2670 & $90^{\circ}$ & $0^{\circ}$ \\
(b) & intermediate & 0.2568 & 1.0875 & 0.7387 & 0.8935 & $81^{\circ}$ & $6^{\circ}$ \\
(c) & high & 0.2945 & 1.0055 & 0.7259 & 0.5288 & $92^{\circ}$ & $21^{\circ}$ \\
\hline
\end{tabular}


Table 2. Additional parameters of configurations of the front of the magnetopause $\left(r_{s}\right.$ has been calculated to satisfy the equilibrium condition, velocities are in $\mathrm{km} / \mathrm{s}$ ).

\begin{tabular}{llccccc}
\hline Case & Latitude & $u_{\infty}$ & $f_{s}$ & $r_{d}$ & $r_{s}$ & $A_{1}$ \\
\hline (a) & low & 500 & 1.2 & 0.1 & 1.81 & 692 \\
(b) & intermediate & 500 & 0.463 & 0.1 & 4.75 & 633 \\
(c) & high & 500 & 0.463 & 0.1 & 7.00 & 589 \\
\hline
\end{tabular}

We have in this case $\mathbf{u}_{2}^{\prime}=0$, then $\mathbf{u}=\mathbf{u}^{\prime}=\mathbf{u}_{1}^{\prime} / 2$, $\varphi_{1}=\psi_{B_{1}, u_{1}^{\prime}}$ and $v^{\prime}=v-u^{\prime} \cos \left(\psi_{1}-\varphi_{1}\right)$. The model provides $\psi_{B_{1}, u_{1}^{\prime}}$, the shear angle $\theta$, the ratio $r_{b}=B_{2} / B_{1}$, as well as the following quantities:

$$
f_{u^{\prime}}=u^{\prime} / u_{\infty} \quad, \quad f_{b}=B_{1} / u_{\infty} \sqrt{4 \pi \rho_{\infty}} \quad, \quad f_{d}=\rho_{1} / \rho_{\infty}
$$

In (??), $u_{\infty}$ and $\rho_{\infty}$ are the velocity and the density of the solar wind, far from the interaction region.

Because the magnetosheath model does not provide magnetospheric values for temperature and density, Table 2 complements the set of parameters needed in the theory. We assume $u_{\infty}=500 \mathrm{~km} / \mathrm{s}$ and use typical values of the density ratio and of the temperatures in both regions, often observed in spacecraft crossings of the magnetopause (expressed as $r_{d}=\rho_{2} / \rho_{1}, r_{s}=S_{2} / S_{1}$ and $\left.f_{s}=S_{1} / u_{\infty}\right)$. Clearly $r_{d}, r_{s}$ and $f_{s}$ must satisfy the equilibrium condition (1). In terms of the $f$ 's and $r$ 's this implies $2 f_{d} f_{s}^{2}\left(1-r_{d} r_{s}^{2}\right)=\gamma f_{b}^{2}\left(r_{b}^{2}-1\right)$. So we first assign $r_{d}$ and $f_{s}$, and then use this relation to calculate $r_{s}$. Since $r_{s}^{2}$ must be positive, we need $f_{s}^{2}>\gamma f_{b}^{2}\left(r_{b}^{2}-1\right) / 2 f_{d}$. When $r_{b}>1$ (as in case (a) below) this condtion sets a lower bound on $f_{s}$.

Using the data of Tables 1 and 2 we then find

$$
u=f_{u^{\prime}} \frac{u_{\infty}}{2}, A_{1}=\frac{u_{1}^{\prime} f_{b}}{f_{u^{\prime}} \sqrt{f_{d}}} \quad, \quad A_{2}=A_{1} r_{b} \sqrt{r_{d}}, S_{1}=\frac{u_{1}^{\prime} f_{s}}{f_{u^{\prime}}} \quad, \quad S_{2}=r_{s} S_{1}
$$

and from these quantities we calculate the remaining parameters of our theory.

\section{Spacecraft data}

At the flanks of the magnetopause, the magnetosheath flow is supersonic and the effects of compressibility on the KHI are expected to be important. We shall examine a configuration at the near equatorial magnetopause at dusk, observed by Interball/tail during the event of January 11, 1997. This was at the tail of a coronal mass ejection passing Earth, during which the dynamic pressure of a high density solar wind strongly compressed the magnetosphere. As a consequence, In- terball/tail was for some time in the magnetosheath, and reentered the magnetosphere, after the dynamic pressure returned to lower values. During the crossing of the magnetopause, from magnetosheath to low latitude boundary layer, the spacecraft measured the physical parameters necessary for a stability analysis. A detailed study of the event is given in ref. [?], together with a discussion of the Kelvin-Helmholtz instability, based on an incompressible model. We intend to revisit that configuration with our compressible theory. The interface that we shall consider is that between the magnetosheath and the low latitude boundary layer.

Table 3. Parameters of the January 11, 1997 event (spacecraft data, velocities are in $\mathrm{km} / \mathrm{s}$ ).

\begin{tabular}{lccllccccc}
\hline Case & $u_{1}^{\prime}$ & $u_{2}^{\prime}$ & $M_{A, 1}$ & $M_{S, 1}$ & $r_{b}$ & $r_{d}$ & $\psi_{B_{1}, u_{1}^{\prime}}$ & $\psi_{B_{1}, u_{2}^{\prime}}$ & $\theta$ \\
\hline (d) & 300 & 105 & 5.0 & 2.0 & 1.5 & 0.117 & $-100^{\circ}$ & $-120^{\circ}$ & $-80^{\circ}$ \\
(e) & 300 & 105 & 5.7 & 2.0 & 1.5 & 0.117 & $-100^{\circ}$ & $-120^{\circ}$ & $-80^{\circ}$ \\
\hline
\end{tabular}

Table 4. Calculated parameters of the January 11, 1997 event (velocities are in $\mathrm{km} / \mathrm{s}$ ).

\begin{tabular}{lcccccc}
\hline Case & $u$ & $\varphi_{1}$ & $u^{\prime}$ & $\delta$ & $r_{s}$ & $A_{1}$ \\
\hline (d) & 102.3 & -89.0 & 200.1 & -15.3 & 2.67 & 60 \\
(e) & 102.3 & -89.9 & 200.1 & -15.3 & 2.67 & 53 \\
\hline
\end{tabular}


In the first line of Table 3 we give the parameters of the event (we use the definitions $M_{A, 1} \equiv A_{1} / u_{1}^{\prime}$, $\left.M_{S, 1} \equiv S_{1} / u_{1}^{\prime}\right)$. The second line contains the parameters of a hypothetical configuration with a slightly larger value of $M_{A, 1}$, to ascertain the effect of this change.

We must recognize that owing to measurement uncertainties, the spacecraft data do not comply with the equilibrium condition (1) at the interface. To emend this flaw, we replaced the measured $r_{s}$ (that is the less reliable datum) by the value derived using the remaining data in the equilibrium condition, expressed as $2 M_{A, 1}^{2}\left(1-r_{d} r_{s}^{2}\right)=\gamma M_{S, 1}^{2}\left(r_{b}^{2}-1\right)$.

The expressions of $u, u^{\prime}, \varphi_{1}, \delta$ and $v^{\prime}$ are given by eqs. (22)-(25), and the remaining quantities we need are:

$$
A_{1}=u_{1}^{\prime} / M_{A, 1}, A_{2}=A_{1} r_{b} \sqrt{r_{d}}, S_{1}=u_{1}^{\prime} / M_{S, 1}, S_{2}=r_{d} S_{1}
$$

We now investigate the stability of these configurations of the magnetopause.

\section{Stability of some magne- topause configurations}

\section{Configurations near the front of the magne- topause}

In the low latitude case (a) there is no magnetic shear, so that the flute modes are unstable, both in the incompressible limit and if compressibility is taken into account. In Fig. 4 we compare the growth rate obtained with the compressible model with that predicted by IMHD (eq. 17c). It can be noticed that there is no significant difference. An increase of the relative velocity by a factor of 3-4 does not change this conclusion.

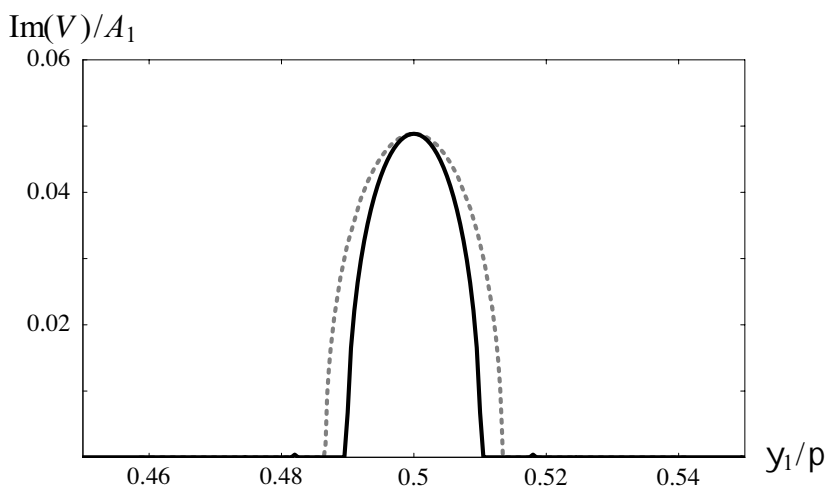

Figure 4. Growth rate of the unstable modes of the low latitude configuration (case (a)). The results of CMHD and IMHD are shown with full lines and gray dashed lines, respectively. The two narrow bands of the secondary instability near $\psi_{1}=0.48 \pi$ and $\psi_{1}=0.52 \pi$ are barely discernible as their growth rates are very small.

The intermediate and high latitude cases are stable when compressibility is taken into account, a result that coincides with the predictions of IMHD. In Fig. 5 we show the stability diagram for the high latitude parameters. We can see the lowering of the critical value $u_{c}$, due to compressibility (already mentioned in Section I). This effect is of no consequence for the configuration (c) owing to the low relative velocity, but it may be relevant for configurations with a large relative velocity (say, $u \approx 1.5 A_{1}$ ).

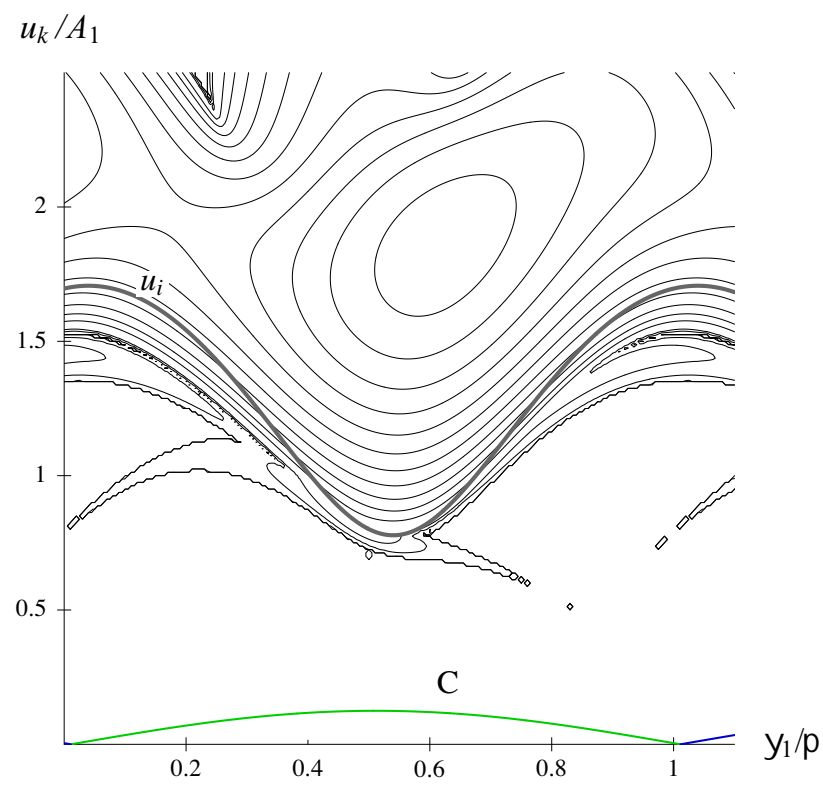

Figure 5. Stability diagram for the parameters of the high latitude case. The curve $\mathrm{C}$ corresponding to the configuration indicates that case $(\mathrm{c})$ is stable.

\section{A configuration in the near flank of the magne- topause}

The stability diagram corresponding to these parameters is shown in Fig. 3. It can be appreciated that this configuration is stable according to the IMHD model, but is unstable when compressibility is taken into account. Moreover, this instability corresponds 
to the secondary modes, which have no counterpart in IMHD. The growth rate of the instability is small, but significant, as can be seen in Fig. 6 .

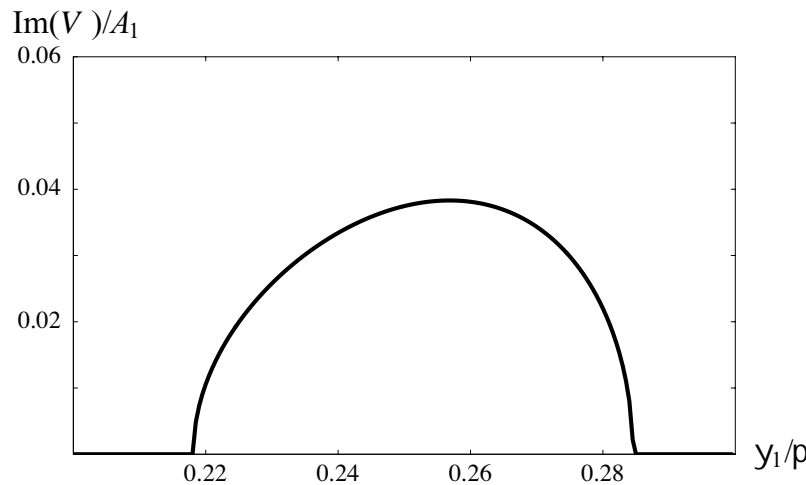

Figure 6. Growth rate of the secondary modes for case (d).

When the line $\mathrm{C}$ of the configuration passes close to the marginal stability line for the primary modes, as happened in this case, the stability properties of the configuration are very sensitive to slight changes of the parameters. This can be appreciated in Fig. 7, in which we show the growth rate of the instability, for a slightly different orientation of the relative velocity $u$ with respect to the magnetic field $\left(\varphi_{1}=75.5^{\circ}\right.$ instead of $89.9^{\circ}$ ). It can be noticed that this change produces a fourfold increase of the growth rate of the secondary instability (and a shift towards larger $\psi_{1}$ ). The primary instability is also present now, although its growth rate is much smaller than that of the secondary modes. This is due to the small- $u_{k}$ destabilization phenomenon, since the modified configuration is still stable according to IMHD.

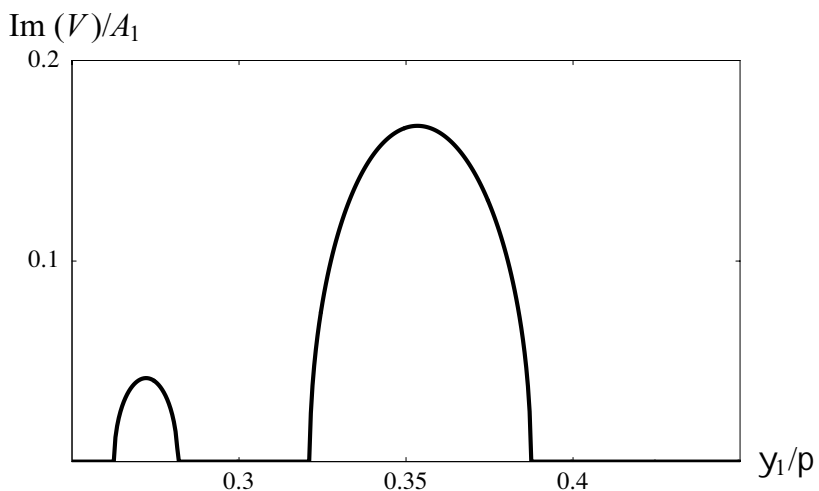

Figure 7. Effect of a change of the orientation of the relative velocity with respect to the magnetic field. The figure represents the growth rate for case $(\mathrm{d})$, with $\varphi_{1}=75.5^{\circ}$ instead of $89.9^{\circ}$. Note the primary modes centered around $\psi_{1} \approx 0.15 \pi$.

If we change the relative velocity, keeping the remaining parameters fixed (as if the solar wind had been slightly faster at that event), the line $\mathrm{C}$ of Fig. 3 shifts upwards, and the primary instability becomes dominant for a $30 \%$ (or larger) increase of $u$.

In Fig. 8 we show the stability diagram for case (e), where $M_{A, 1}$ differs from case (d) by $14 \%$. The effect of this change is to increase the growth rate of the secondary instability with respect to the results of Fig. 6. This modified configuration is stable according to IMHD.

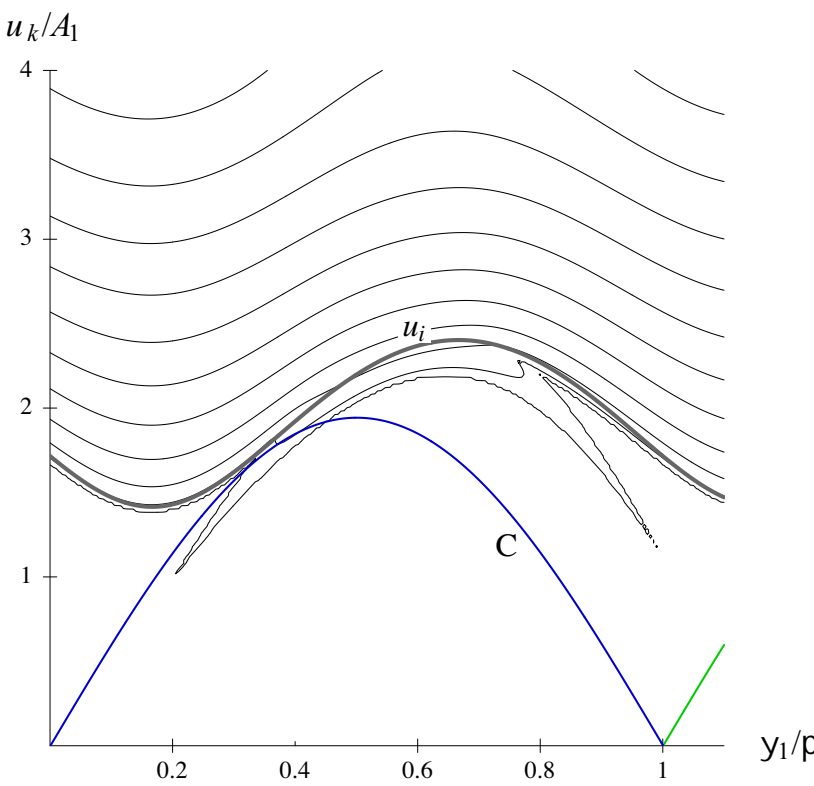

Figure 8. Stability diagram for the parameters of the event of January 11, 1997. The curve C corresponds to the configuration of case (e).

\section{Conclusions}

Compressibility affects the Kelvin-Helmholtz instability by changing the unstable domains of the modes already present in the incompressible model as well as their growth rate. Modes with large $u_{k}$ (the projection of the relative velocity on the wave vector of the perturbation) are stabilized. At the same time the lower boundary of the unstable $u_{k}$ interval shifts downwards, thus destabilizing perturbations that are stable according to IMHD, for $u_{k}<u_{i}$ (the critical value for marginal incompressible instability). On the other hand, the growth rate of the unstable modes above $u_{i}$ is reduced by compressibility. In addition, compressibility introduces new unstable modes (the secondary modes) that do not exist in IMHD, and that occur for $u_{k}$ below $u_{i}$. For these reasons, compressibility may play an important role in determining the stability of configurations with a relative velocity of the order of $u_{i}$.

For configurations of the front of the magnetopause, which have small relative velocities, the IMHD model gives reliable estimates of their stability, and compressibility effects do not introduce significant changes. However, compressibility must be taken into account when assessing the stability properties of configurations 
at the flanks of the magnetopause. As shown in Section VII, in these cases the occurrence of the secondary instability and the shift of the boundary of the primary instability play an important role. Then, configurations that are stable if compressibility is neglected turn out to be unstable when it is considered. In these situations, the stability properties are quite sensitive on the values of the parameters. The estimates based on IMHD may be misleading, and a careful analysis is required in each case, since no simple rule of thumb can be given. In these respects the stability diagrams introduced in Section V may prove useful, since a single graph allows to visualize the effect of changing the magnitude and orientation of the relative velocity.

\section{Acknowledgments}

We acknowledge grants from CONICET (PIP 4521/96 and PIP 4535/96), the University of Buenos Aires (Projects X151 and TX32) and NASA (grant NAG5-2834).

\section{References}

[1] M. G. Kivelson and S. H. Chen. The magnetopause: Surface waves and instabilities and their possible dynamic consequences. AGU Monograph 90, Physics of Magnetopause, edited by P. Song, B. U. O. Sonnerup and M. F. Thomsen. Geophys. Mon. Ser. 90, AGU, Washington, DC, 257 (1995).

[2] A. Miura. Kelvin-Helmholtz instability at the magnetopause: computer simulations. AGU Monograph 90, Physics of Magnetopause, edited by P. Song, B. U. O. Sonnerup, and M. F. Thomsen. Geophys. Mon. Ser. 90, AGU, Washington, DC, 285 (1995).

[3] C. J. Farrugia, F. T. Gratton, and R. B. Torbert. Viscous-type processes in the solar windmagnetosphere interaction. Special volume Challenge to Long-Standing Unsolved Space Physics Problems in the 20th Century. Space Science Review, 95 (1/2), 443 (2001).

[4] S. Duhau and J. Gratton. Kelvin-Helmholtz instability of anisotropic plasma in a magnetic field. J. Plasma Phys. 13, 451 (1975).

[5] F. Gratton, J. Gratton, and A. G. González. Convective instability of internal modes in accelerated compressible plasmas. Plasma Phys. Controlled Fusion 30, 435 (1988).

[6] W. A. Newcomb. Compressibility effects on instability growth rates. Phys. Fluids 26, 3246 (1983).

[7] M. G. Kivelson and D. J. Southwood. Coupling of global magnetospheric MHD eigenmodes to field line resonances. J. Geophys. Res. 91, 4345 (1986).

[8] M. G. Kivelson and Z.-Y. Pu. The Kelvin-Helmholtz instability on the magnetopause. Planet. Space. Sci. 32, 1335 (1984).

[9] A. G. González and J. Gratton. The Kelvin-Helmholtz instability in compressible plasmas, in Proc. VIII Latin-American Workshop on Plasma Physics. Vol. 1 (Review papers) p. 23. Tandil, Argentina (1998).
[10] A. Miura and P. L. Pritchett P. Nonlocal stability analysis of the MHD Kelvin-Helmholtz instability in a compressible plasma. J. Geophys. Res. 87, 7431 (1982).

[11] C. J. Farrugia, F. T. Gratton, L. Bender, J. M. Quinn, R. B. Torbert, N. V. Erkaev and H. K. Biernat. Recent work on the Kelvin-Helmholtz instability at the dayside magnetopause and boundary layer. Polar Cap Boundary Phenomena, edited by J. Moen et al., Kluwer Academic Publishers, Netherlands, 1 (1998).

[12] A. G. González and J. Gratton. The Kelvin-Helmholtz instability in a compressible plasma: the role of the orientation of the magnetic field with respect to the flow. J. Plasma Phys. 51, 43 (1994).

[13] A. G. González and J. Gratton. The role of a density jump in the Kelvin-Helmholtz instability of a compressible plasma. J. Plasma Phys. 52, 233 (1994).

[14] A. G. González, J. Gratton and M. González. The Kelvin-Helmholtz instability in compressible plasmas with magnetic field shear. Proc. IX LAWPP, AIP Conf. Proc. 563, 47 (2001).

[15] S. Chandrasekhar. Hydrodynamic and Hydromagnetic Stability. Oxford Univ. Press (1961).

[16] W. I. Axford. Quart. J. Mech. Appl. Math. 13, 314 (1960).

[17] W. I. Axford. Can. J. Phys. 40, 654 (1962).

[18] D. J. Southwood. The hydrodynamic stability of the magnetospheric boundary. Planet. Space Sci. 16, 587 (1968).

[19] A. Miura. Dependence of the magnetopause KelvinHelmholtz instability on the orientation of the magnetosheath magnetic field. Geophys. Res. Lett. 22, 2993 (1995).

[20] C. J. Farrugia, F. T. Gratton, L. Bender, H. K. Biernat, N. V. Erkaev, J. M. Quinn, R. B. Torbert and V. Dennisenko. Charts of joint Kelvin-Helmholtz and Rayleigh-Taylor instabilities at the dayside magnetopause for strongly northward interplanetary magnetic field. J. Geophys. Res. 103, 6703 (1998).

[21] J. E. Contín, F. T. Gratton and C. J. Farrugia. A study of Kelvin-Helmholtz modes for hyperbolic tangent field profiles applied to the dayside magnetopause. Fluidos 2001, edited by J. C. Ferreri. ISBN 927-201480-9 (2001).

[22] J. E. Contín. Ph. D. Thesis, Inestabilidades de láminas de corriente con flujos de plasma y aplicaciones a la magnetopausa diurna. Facultad de Ciencias Exactas y Naturales, Universidad de Buenos Aires (1999).

[23] N. V. Erkaev. Results of the investigation of MHD flow around the magnetosphere. Geomagn. Aeron. 28, 455 (1988).

[24] C. J. Farrugia, F. T. Gratton, J. Contin, C. C. Cocheci, R. L. Arnoldy, K. W. Ogilvie, R. P. Lepping, G. N. Zastenker, M. N. Nozdrachev, A. Fedorov, J.-A. Sauvaud, J. T. Steinberg, and G. Rostoker. Coordinated Wind, Interball/tail, and ground observations of Kelvin-Helmholtz waves at the near-tail, equatorial magnetopause at dusk: January 11, 1997. J. Geophys. Res. A4, 105, 7639 (2000). 